\title{
Are Changes in Ocular Aberrations With Age a Significant Problem for Refractive Surgery?
}

\author{
Susana Marcos, $\mathrm{PhD}$
}

\begin{abstract}
We discuss current knowledge about the change of aberrations with aging, cataract surgery, and laser in situ keratomileusis (LASIK) for myopia. Based on this evidence, we speculate about the long-term expectations for postoperative LASIK eyes in terms of aberrations. Standard myopic LASIK surgery produces a significant increase in aberrations, particularly corneal spherical aberration, which changes to positive values. Aberrations increase with age, and in particular, the spherical aberration of the crystalline lens shifts toward positive values. Therefore, no compensatory effect is expected to occur with age after standard myopic LASIK, but rather the unusually high amount of aberrations in postoperative LASIK patients is expected to worsen with age. The amount of aberrations in patients after cataract surgery with implantation of standard intraocular lenses (IOLs) is higher than in normal young subjects. If an ideal customized ablation (not inducing aberrations and reducing naturally existing aberrations) is ever possible, the perfect correction will not last (due to the change of aberrations with age), and aberrations of the crystalline lens corrected on the cornea are likely to reappear after conventional cataract surgery. Potential benefits of customized IOLs for cataract surgery and improved optics in older patients are discussed. [J Refract Surg 2002; 18:Sxxx-Sxxx]
\end{abstract}

From the Instituto de Optica, Consejo Superior de Investigaciones Científicas, Madrid, Spain.

The author acknowledges the co-authors of the published results reviewed here: James Maclellan and Stephen A. Burns (Schepens Eye Research Institute, Harvard University), Sergio Barbero and Lourdes Llorente (Instituto de Optica, Consejo Superior de Investigaciones Científicas), and collaborative surgeons Jesús Merayo-Lloves (Instituto de Oftalmobiología Aplicada, Universidad de Valladolid- Unidad Asociada IO/CSIC-IOBA/UVA) and Ignacio Jiménez-Alfaro (Fundación Jiménez Díaz). The author ackowledges support of Carl-Zeiss Spain for the loan of an Atlas Humphrey Corneal Topography System and partial funding of a research fellowship; and CAM08.7/0010.1/2000 and Convenio de Cooperacion USA-Spain for research funding.

The author has no proprietary interest in the materials herein.

Presented at the 3rd International Congress of Wavefront Sensing and Aberration-free Refractive Correction, February 15-17, 2002, Interlaken, Switzerland.

Correspondence: Susana Marcos, PhD, Serrano 121, 28006 Madrid, Spain. Tel: 34.915616800 x 2306; Fax: 34. 915645557; E-mail: susana@io.cfmac.csic.es
W ith the increasing popularity of laser in situ keratomileusis (LASIK) for treatment of refractive errors (myopia in particular), it is expected that in a few decades a significant portion of the aging population will have undergone LASIK at an earlier age. Morgan ${ }^{1}$ predicted, concerning the age-related shift toward hyperopia in late adulthood, that there may be "a number of very unhappy senior citizens who had successful refractive surgery when young adults," since mild myopia reduces the dependence on reading glasses. An additional well-known problem appears in the computation of IOL power for cataract surgery in patients who have undergone a keratorefractive surgical procedure, particularly if keratometry before LASIK, radial keratometry (RK), or photorefractive keratectomy (PRK) is not available. ${ }^{2}$

Although these concerns refer to major refractive error (defocus), high order aberrations also play a role in the subject's quality of vision. It is well accepted that aberrations change with age. ${ }^{3-6}$ Further changes occur with the replacement of the crystalline lens by an artificial IOL during cataract surgery. $^{7-9}$ On the other hand, refractive surgery modifies the aberration pattern. ${ }^{10-13}$ A review follows about how aberrations change with LASIK for myopia, aging, and cataract surgery, and how the natural increase of aberrations with aging may be aggravated in eyes that have had LASIK for myopia.

\section{EVIDENCE}

Change of Aberrations With Age

Contrast sensitivity decreases with age.14,15 Although some of these changes are neural, an important part of contrast sensitivity loss is due to degradation of the eye's optical quality. Decrease in the modulation transfer function that occurs with age $^{5}$ is partly due to an increase of intraocular scattering ${ }^{16}$, and an increase of optical aberrations..$^{3,4,6}$ McLellan et $\mathrm{al}^{3}$ did a cross-sectional study on 


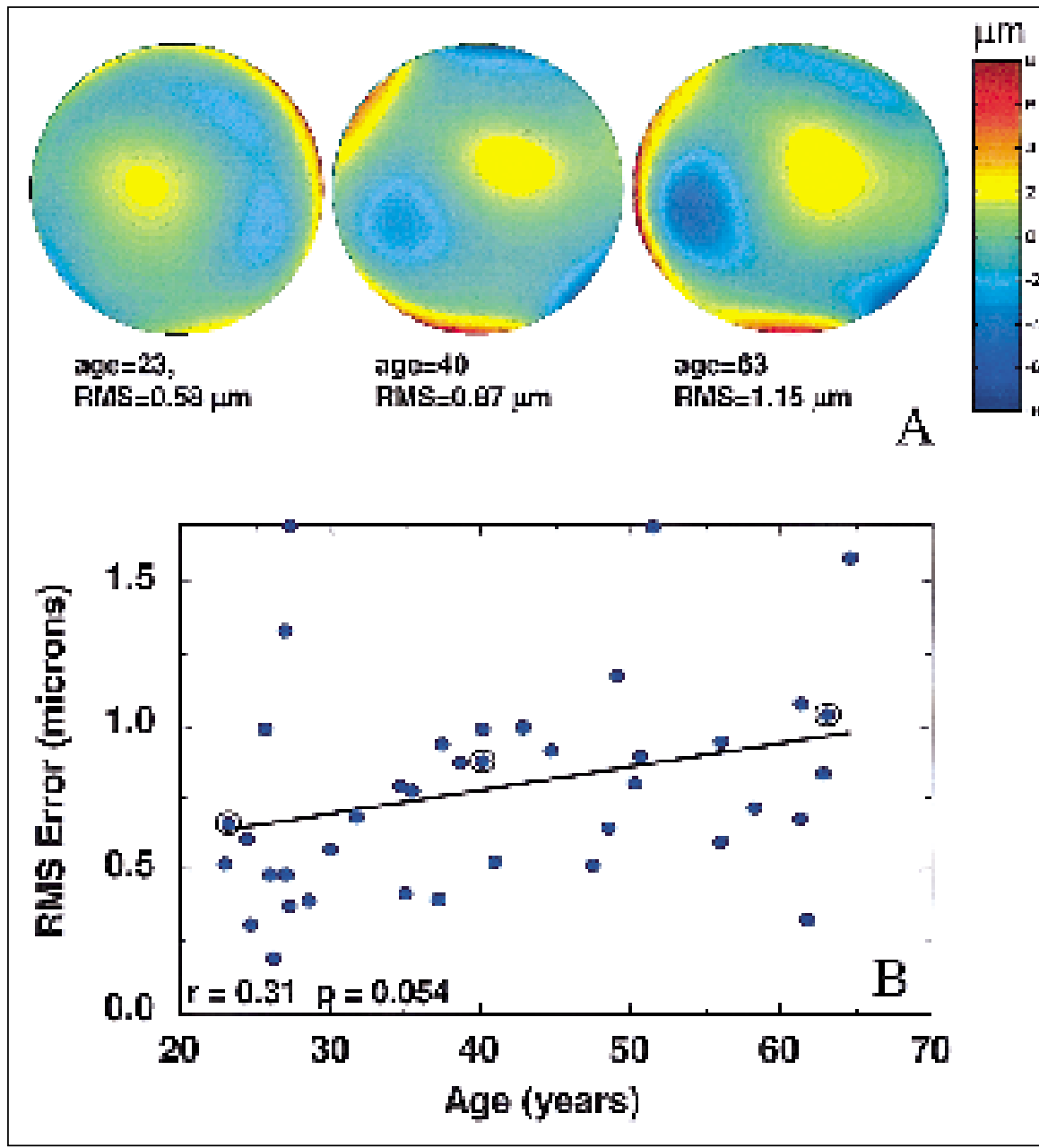

Figure 1. A) Examples of total wave aberrations for three subjects of different ages, measured using the Spatially Resolved Refractometer (Schepens Eye Research Institute, Boston, MA), for third and higher order aberrations. Pupil diameter $=7.32 \mathrm{~mm}$. B) Root mean square (RMS) wavefront error for 3rd and higher order aberrations as a function of age (pupil diameter=7.32 mm). The solid line represents a linear regression to the data. Circled points correspond to the three subjects of Figure 1A. Data from McLellan et al. $^{3}$

40 normal subjects (age range from 21 to $65 \mathrm{yr}$ ) using the Spatially Resolved Refractometer (developed at the Schepens Eye Research Institute ${ }^{17,18}$ ), and showed that non-conventional aberrations increased statistically significantly with age. Figure 1B shows the correlation between high order root mean square wavefront error (RMS, a wave aberration-based optical quality metric) and age. Figure $1 \mathrm{~A}$ shows three wave aberration maps of eyes with RMS values close to the regression line. ${ }^{3}$ Although a non-significant increase was found for $3 r d$ order aberrations, 4th order aberrations (ie, spherical aberration) and higher order terms increased statistically significantly with age. ${ }^{3}$ Artal and colleagues' cross-sectional study ${ }^{4}$ on 17 subjects (age range from 26 to $69 \mathrm{yr}$ ) using a Shack-Hartmann system (developed at the University of Murcia) also reported significant increase in the amount of $3 \mathrm{rd}$ and higher order ocular aberrations with age. Where do these optical changes occur? Minor changes are expected to occur on the cornea. Corneal astigmatism has been shown to change from with-therule to against-the rule with age, and an increase of prevalence of astigmatism with age has been reported. ${ }^{19}$ Oshika and colleagues ${ }^{20}$ found a significant increase in 3rd order corneal aberration terms only, and Guirao and colleagues ${ }^{20}$ found that spherical aberration was also slightly larger for ol der corneas.

Corneal changes alone are not sufficient to explain the increase of ocular aberrations with age. The crystalline lens thickens throughout life. ${ }^{22}$ In addition, the anterior lens surface has been reported to become flatter with age 23-25, although some of these results may be slightly biased by artifacts of the Scheimpflug technique used to image the anterior segment. ${ }^{25}$ Changes in the refractive index in the cortex of the lens ${ }^{26,27}$ have been hypothesized to compensate for the increased lens power that should result from the decrease in radii of curvature with age. Such a compensatory mechanism is postulated, since a myopic shift is not observed with age (lens paradox). Measurements on post mortem crystalline lenses $^{28}$ and eye models based on geometric and optical properties of the aging crystalline lens ${ }^{29}$ 


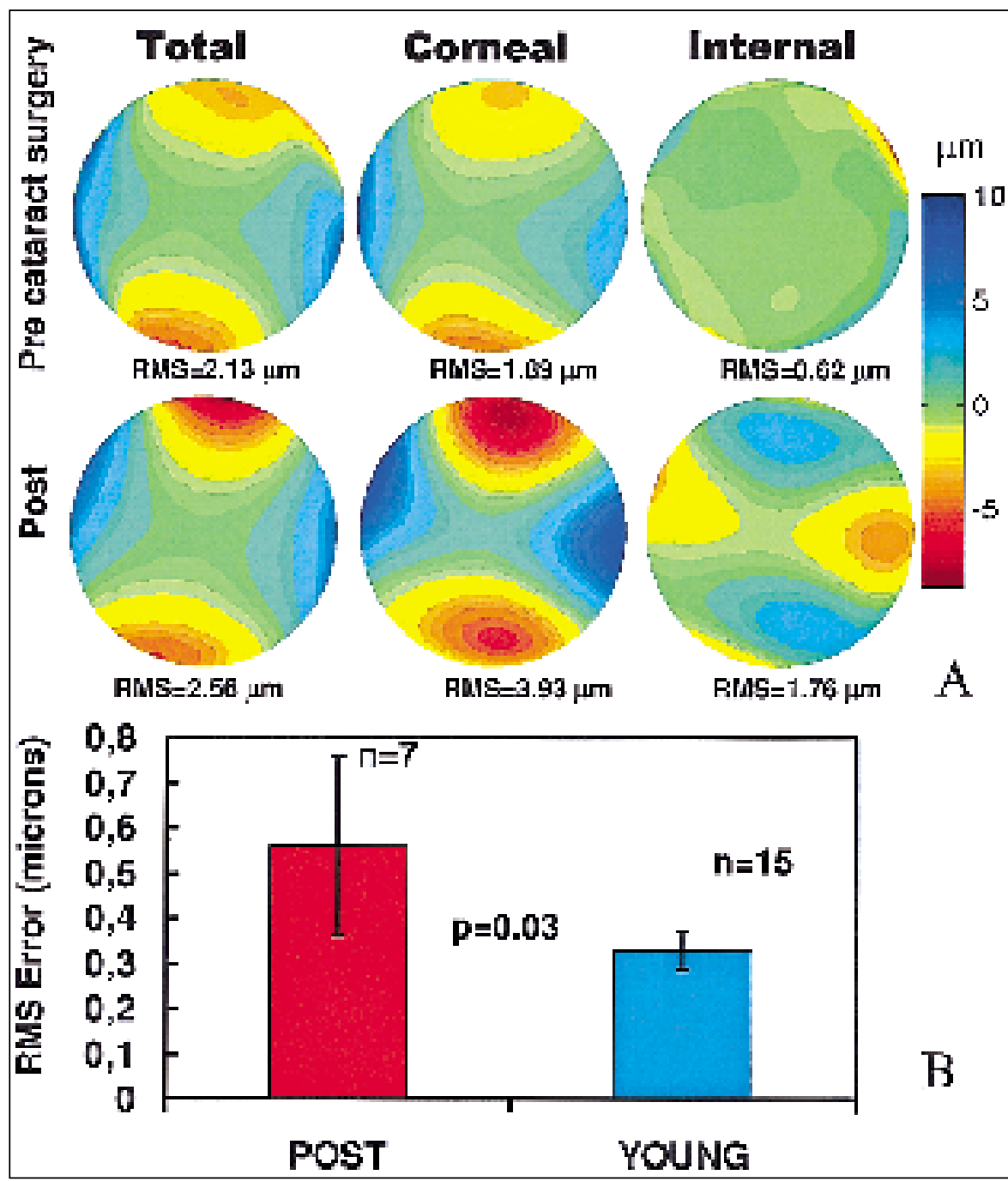

Figure 2. A) Example of total, corneal, and internal wave aberrations for one subject (age $71 \mathrm{yr}$ ) before and after cataract surgery (phacoemulsification) and IOL implantion (Acrysof, Alcon), canceling tilt and defocus. Total aberrations were measured with laser ray tracing and corneal aberrations with an Atlas Humphrey (Carl Zeiss) videokeratoscope and custom algorithms. Pupil diameter $=5 \mathrm{~mm}$. B) RMS wavefront error for 3rd and higher order internal aberrations (pupil diameter $=$ $5.5 \mathrm{~mm}$ ). The left bar represents the average internal RMS for postoperative cataract eyes (accounting mostly for the contribution of the $\mathrm{IOL}$ ) and the right bar represents the average internal RMS (crystalline lens) for a group of young eyes. Error bars indicate standard deviation. Data from Barbero et al. ${ }^{8}$

conclude that generally negative spherical aberration of the crystalline lens shifts toward positive values with age. This agrees with ocular aberration measurements, which also show a shift of spherical aberrations in the positive direction. Finally, by measuring corneal and total aberration in the same group of 17 eyes, Artal and colleagues ${ }^{4}$ reported a loss of balance of the aberrations of the individual ocular components with age, including comatic terms.

\section{Change of Aberrations With Cataract Surgery}

Almost $90 \%$ of the population over 75 years of age develops a cataract, which is typically uneventfully replaced by an artificial intraocular lens implant. The first in vivo assessment of optical quality with intraocular lenses was done using a double-pass technique at the Instituto de Optica (CSIC), Madrid, Spain. ${ }^{9}$ Although patients experienced a dramatic improvement in contrast sensitivity (greatly degraded by the scattering produced by the cataract), the modulation transfer function (MTF) in patients after successful cataract surgery was significantly lower than the MTF in normal, young subjects. Mierdel and colleagues ${ }^{7}$ measured high order aberrations in a group of 10 patients after cataract surgery. Although they did not find abnormally large aberrations, high order coefficients showed high variability in this group of patients. Preliminary results ${ }^{8}$ from our laboratory (Institute of Optics CSIC, Madrid) measuring corneal aberrations (using custom algorithms to process corneal elevation maps recorded with an Atlas Humphrey videokeratoscope ${ }^{10,30,31}$ ) and total aberrations (using a laser ray tracing system ${ }^{32,33}$ ) after cataract surgery (by phacoemulsification) show changes in both corneal and total aberrations. Reliable preoperative measurements (through the cataract) were possible in some, but not all patients. Figure 2A shows corneal, total, and internal (total minus 


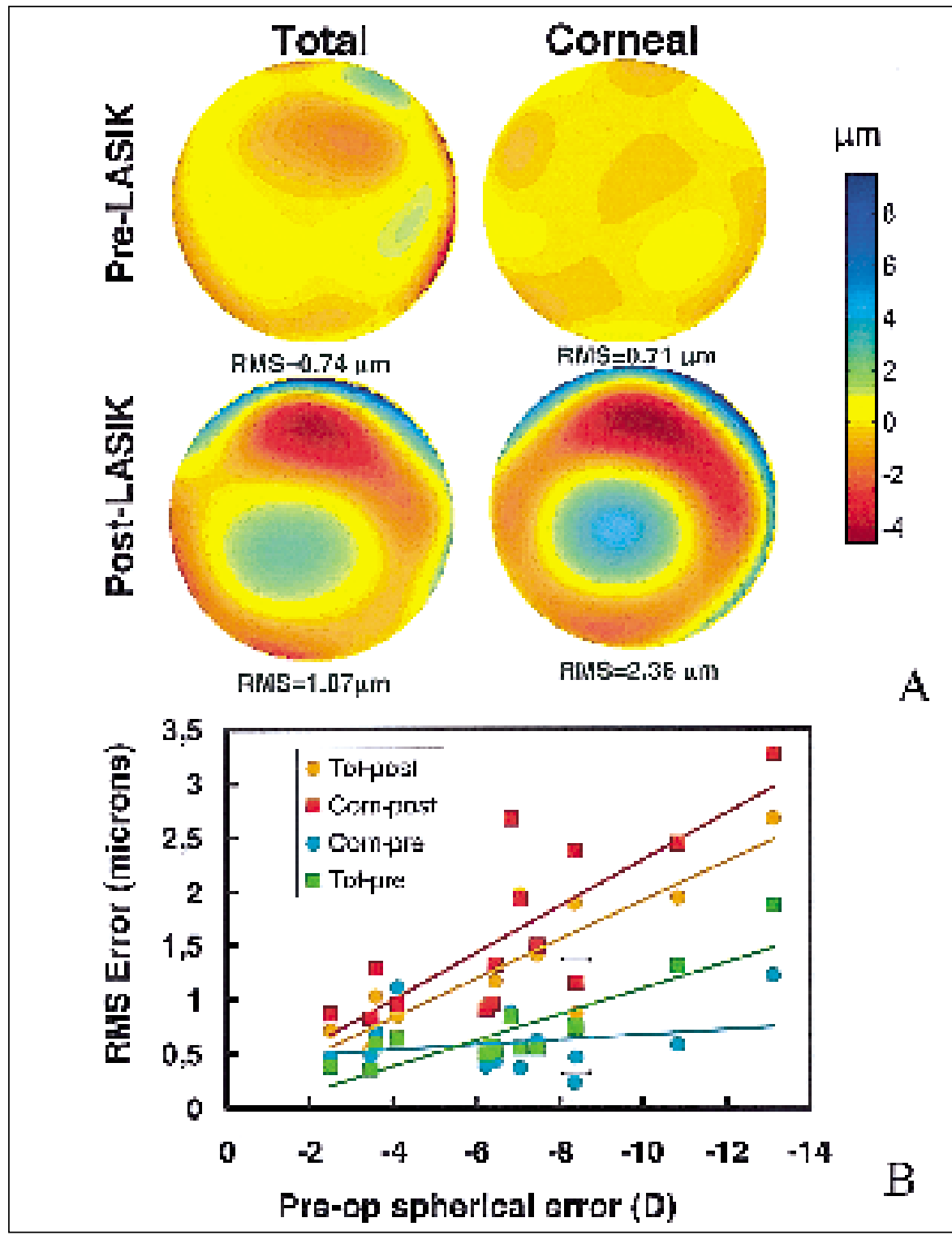

Figure 3. A) Example of total and corneal aberration before and after standard LASIK for myopia, for 3rd and higher order aberrations (pupil diameter=6.5 mm). B) RMS wavefront error for 3rd and higher order aberrations for corneal (squares) and total (circles) aberrations, before (green and blue, respectively) and after LASIK (red and orange, respectively). Pupil diameter $=6.5 \mathrm{~mm}$. Points are plotted as a function of preoperative spherical error. Circled points correspond to the eye shown in Figure 3A. Data from Marcos et al. ${ }^{10}$

corneal) wave aberration maps for a typical patient, before and after IOL (Acrysof, Alcon) implant. An increase in corneal (and total) astigmatism is observed, attributed in the literature to the effect of the incision. ${ }^{34}$ Changes in corneal high order aberration terms were also found in some subjects. Figure 2B shows internal RMS (3rd and higher order aberrations) after cataract surgery in a group of seven patients (mean age $75 \pm 4.8 \mathrm{yr}$ ), in comparison with a group of young, normal subjects (mean age $29 \pm 3.7 \mathrm{yr}$, spherical error <4.50 diopters [D]). A recent study by Barbero and colleagues ${ }^{31}$ measuring anterior corneal and total aberrations in an aphakic eye, suggested a negligible contribution of the posterior corneal surface after lens extraction, as is presumably the case in normal eyes. If so, the average internal RMSs plotted in Figure $2 \mathrm{~B}$ is mainly due to aberrations of the intraocular lens (Acrysof in all patients) and aberrations of the young crystalline lens, respectively. We found that the internal RMS after cataract surgery is only slightly lower (not statistically significant) than the RMS of the crystalline lens with the cataract. ${ }^{8} \mathrm{Also}$, although the spherical aberration of the crystalline lens tended to be negative in young eyes, the spherical aberration of the IOL was al ways positive. This measure of 3rd through 7th aberration orders accounts for the geometric properties of the lens, and does not include high order irregularities and scattering, which are eliminated with the IOL implant. In summary, cataract surgery modifies the aberration pattern, both the corneal and especially 
the internal aberrations. The increase of aberration in postoperative cataract surgery eyes with respect to young eyes is largely due to aberrations of the IOL, loss of balance of internal and corneal spherical aberration (both positive after cataract surgery), and tilt and centration of the IOL. Individual variations of positioning errors and differences in IOL power may explain the variability found in Figure 2B. Current procedures do not fully restore optical quality values to those of young subjects.

\section{Change of Aberrations With Refractive Surgery}

In LASIK for myopia, the central part of the cornea is flattened to decrease its power. ${ }^{35}$ This produces a change in corneal asphericity resulting in an increase in corneal spherical aberration toward more positive values. ${ }^{36}$ In a recent study, we measured both corneal and total aberrations in a group of 14 eyes before and after (at least 1 month) LASIK for myopia (mean age $28.9 \pm 5.4 \mathrm{yr}$; mean preoperative spherical error, $-6.80 \pm 2.90 \mathrm{D}) .{ }^{10}$ Third and higher order aberrations increased significantly after LASI K, the larger the correction the higher the increase. The most important change occurred for spherical aberration, although 3rd order terms also increased significantly. Most of the optical changes can be accounted for by the anterior corneal aberrations. Figure $3 \mathrm{~A}$ shows corneal and total aberration maps (3rd order aberration and higher), preoperative, and postoperative LASI K, for one eye (preoperative spherical error, $-8.40 \mathrm{D}$ ). Figure 3B shows RMS (for both total and corneal aberrations, before and after LASIK) as a function of preoperative spherical error, for a group of 14 eyes. The preoperative amount of 3rd and higher order aberrations is correlated with the amount of myopia, for the total, but not significantly for corneal aberrations. ${ }^{37}$ The induced spherical aberration is slightly higher for the total than for the anterior corneal aberrations, and both corneal and total aberrations after LASIK are highly correlated with the amount of preoperative spherical error. The observed changes in total optical quality correlate well with visual performance. ${ }^{38}$ Most of the decrease in contrast sensitivity in these patients could be attributed to a decrease in the MTF (computed from the total wave aberration). Current efforts in the refractive surgery field aim at compensating not only 2 nd order aberrations (defocus and astigmatism), but also higher order terms, to sculpt the cornea in such a way that aberrations of the eye are minimized. ${ }^{39}$

\section{DISCUSSION}

Until data become available on the change of aberrations with aging in patients who have undergone refractive surgery, we need to base our predictions on the evidence presented in the previous section. LASIK for myopia increases the spherical aberration of the cornea toward positive values. The spherical aberration of the crystalline lens shifts toward positive values with aging, so no compensatory effect is expected to occur with aging; on the contrary, the adverse effects of an increased spherical aberration are expected to worsen with age. Corneal changes are minor with normal aging. It remains to be seen whether larger changes may occur in thinner, postoperative corneas. Cataract surgery modifies the aberration pattern. The intraocular lenses measured in this study showed positive spherical aberration, and significant amounts of 3rd order aberration (possibly associated with decentrations). IOLs, although they suppress scattering, do not restore optical quality to young subjects' levels. Therefore, higher aberrations after cataract surgery are expected to be much higher in patients who have had LASIK for myopia than for the normal elderly subject. In particular, the abnormally high positive spherical aberration of the postoperative LASI K cornea will add up to the positive spherical aberration of the IOL. If custom ablation becomes a reality, it might be possible that the described problems inherent to current procedures (increase of high order aberrations) are attenuated. In fact, current efforts aim at canceling not only 2 nd order aberrations, but also naturally occurring high order aberrations during the surgical procedure. However, given the changes of aberrations with age, this perfect correction will not last forever. Also, if aberrations of the crystalline lens have been corrected by modifying the corneal shape, those are likely to reappear when the lens is replaced by a conventional IOL.

The impact of some of the mentioned problems associated with an increase of aberrations may be attenuated by pupillary miosis ${ }^{6}$, the decreased natural pupil size with age. In addition, a larger amount of aberrations results in larger depth-offiel $\mathrm{d}^{40}$, which may help to gain some multifocality in eyes with no accommodation capability. Also, some of the mentioned problems may be resolved if optimized aspheric IOLs were implanted at the time of cataract surgery to compensate for the increased positive spherical aberration of the anterior cornea 
in postoperative LASIK patients. Accurate positioning of these lenses is critical to achieve the expected benefit. Atchison ${ }^{41}$ concluded that if these lenses are not well centered, the asphericity that eliminates spherical aberration may result in poorer performance than that occurring with spherical surfaces. In any case, even if an ideal compensation of aberrations was possible in the elderly eye, it is questionable that this may be beneficial. Perfect optical systems have a much narrower depth of field than aberrated systems, and for a certain range of focus, absolute defocused performance can be even worse. However, tolerance to defocus is desirable in eyes that lack an accommodation response. An increase in optical contrast may help eyes with reduced visual sensitivity from neural factors. ${ }^{42}$ However, the loss of photoreceptors occurring with aging (although the decline is more marked for the rod than for cones) 43,44 , or the degradation of visual pathways critical for visual acuity ${ }^{45}$, makes it unlikely that those eyes can fully benefit from the potential increased spatial resolution provided by optimized optics.

\section{REFERENCES}

1. Grosvenor T, Goss DA. Clinical Management of Myopia. Philadel phia, PA: Butterworth-Heinemann; 1999.

2. Seitz B, Langenbucher A. Intraocular lens power calculation in eyes after corneal refractive surgery. J Refract Surg 2000;16:349-361.

3. Mclellan J, Marcos S, Burns S. Age-related changes in monochromatic wave aberrations in the human eye. Invest Ophthalmol Vis Sci 2001;42:1390-1395.

4. Artal P, Berrio E, Guirao A, Piers P. Contribution of the cornea and internal surfaces to the change of ocular aberrations with age. J Opt Soc Am A 2002;19:137-143.

5. Artal P, Ferro M, Miranda I, Navarro R. Effects of aging in retinal image quality. J Opt Soc Am A 1993;10:1656-1662.

6. Calver R, Cox M, Elliott D. Effect of aging on the monochromatic aberrations of the human eye. J Opt Soc Am A 1999;16:2069-2078.

7. Mierdel $P$, Kaemmerer $M$, Krinke HE, Seiler T. Effects of photorefractive keratectomy and cataract surgery on ocular optical errors of higher order. Graefe's Arch Clin Exp Opthalmol 1999;237:725-729.

8. Barbero S, Marcos S, Llorente L. Optical changes in corneal and internal optics with cataract surgery [ARVO abstract]. Available at: http://www.arvo.org. Access confirmed J uly 23, 2002.

9. Artal P, Marcos S, Navarro R, Miranda I, Ferro M. Throughfocus image quality of eyes implanted with monofocal and multifocal intraocular lenses. Optical Engineering 1995;34:772-779.

10. Marcos S, Barbero B, Llorente L, Merayo-Lloves J. Optical response to LASIK for myopia from total and corneal aberrations. Invest Ophthalmol Vis Sci 2001:42:3349-3356.

11. Moreno-Barriuso E, Merayo-Lloves ], Marcos S, Navarro R, Llorente L, Barbero S. Ocular aberrations before and after myopic corneal refractive surgery: LASIK-induced changes measured with laser ray tracing. Invest Ophthalmol Vis Sci 2001;42:1396-1403.

12. Kaemmerer $M$, Mrochen $M$, Mierdel $M$, Seiler T. High order aberrations induced by photorefractive keratectomy (PRK) and LASIK. Invest Ophthalmol Vis Sci 1999;40(suppl):7.

13. Oshika T, Klyce SD, Applegate RA, Howland HC, EI Danasoury MA. Comparison of corneal wavefront aberrations after photorefractive keratectomy and laser in situ keratomileusis. Am J Ophthalmol 1999;127:1-7.

14. Elliott D, Whitaker D, MacVeigh D. Neural contribution to spatiotemporal contrast sensitivity decline in healthy ageing eyes. Vision Res 1990;30:541-547.

15. Owsley C, Sekuler R, Siemsen D. Contrast sensitivity throughout adulthood. Vision Res 1983;23:689-699.

16. Westheimer G, Liang J . Influence of ocular light scatter on the eye's optical performance. J Opt Soc Am A 1995;16: $1417-1424$.

17. Webb RH, Penney CM, Thompson KP. Measurement of ocular wavefront distortion with a spatially resolved refractometer. Applied Optics 1992;31:3678-3686.

18. He J C, Marcos S, Webb RH, Burns SA. Measurement of the wave-front aberration of the eye by a fast psychophysical procedure. J Opt Soc Am A 1998;15:2449-2456.

19. Pierscionek BK. Aging changes in the optical elements of the eye. J Biomed Opt 1996;1:147-157.

20. Oshika T, Klyce SD, Applegate RA, Howland HC. Changes in corneal wavefront aberrations with aging. Invest Ophthalmol Vis Sci 1999;40:1351-1355.

21. Guirao A, Redondo M, Artal P. Optical aberrations of the human cornea as a function of age. J Opt Soc Am A 2000;17:1697-1702.

22. Dubbelman $M$, van der Heijde $G$, Weeber $H$. The thickness of the aging human lens obtained from corrected Scheimpflug images. Optom Vis Sci 2001;78:411-416.

23. Koretz J , Cook C, Kaufman P. Aging of the human lens: changes in lens shape upon accommodation and with accommodative loss. J Opt Soc Am A 2002;19:144-151.

24. Brown N. The change in lens curvature with age. Exp Eye Res 1974;19:175-183.

25. Dubbelman $M$, van der Heijde. The shape of the aging human lens: curvature, equivalent refractive index and the lens paradox. Vision Res 2001;41:1867-1877.

26. Smith G, Atchison D, Pierscionek B. Modeling the power of the aging human eye. J Opt Soc Am A 1992;9:2111-2117.

27. Hemenger $H$, Garner $L$, Ooi $S$. Change with age of the refractive index gradient of the human ocular lens. Invest Ophthalmol Vis Sci 1995;36:703-707.

28. Glasser A, Campbell M. Biometric, optical and physical changes in the isolated human crystalline lens with age in relation to presbyopia. Vision Res 1999;39:1991-2015.

29. Smith $G$, Atchison $D$. The gradient index and spherical aberration of the lens of the human eye. Ophthalmology Physiological Optics 2001;21:317-326.

30. Barbero S, Marcos S, Merayo-Lloves J , Moreno-Barriuso E. Validation of the estimation of corneal aberrations from videokeratography in keratoconus eyes. J Refract Surg 2002;18:263-270.

31. Barbero S, Marcos S, Merayo-Lloves J M. Exploring total and corneal aberrations in an unilateral aphakic subject. J Cataract Refract Surg, in press.

32. Navarro R, Losada MA. Aberrations and relative efficiency of light pencils in the living human eye. Optom Vis Sci 1997;74:540-547.

33. Moreno-Barriuso E, Marcos S, Navarro R, Burns SA. Comparing laser ray tracing, spatially resolved refractometer and Hartmann-Shack sensor to measure the ocular wavefront aberration. Optom Vis Sci 2001;78:152-156.

34. J acobs B, Gaynes BL, Deutsch TA. Refractive astigmatism after oblique clear corneal phacoemulsification cataract incision. J Cataract Refract Surg 1999;25:949-952.

35. Pallikaris I, Papatzanaki M, Stathi E, Frenschock O, Georgiadis A. Laser in situ keratomileusis. Lasers Surg Med 1990;10:463-468. 
36. Holladay J T, Dudeja DR, Chang J. Functional vision and corneal changes after laser in situ keratomileusis determined by contrast sensitivity, glare testing and corneal topography. J Cataract Refract Surg 1999;25:663-669.

37. Marcos S, Barbero S, Llorente L, Why high myopic eyes tend to be more aberrated? Optical Society of America Technical Digest 2001:75.

38. Marcos S. Aberrations and visual performance following standard laser vision correction. J Refract Surg 2001;17:596-601.

39. MacRae SM, Schwiegerling J , Snyder R. Customized corneal ablation and super vision. J Refract Surg 2000;16:230-235.

40. Marcos S, Moreno E, Navarro R. The depth-of-field of the human eye from objective and subjective measurements. Vis
Res 1999;39:2039-2049.

41. Atchison DA. Design of aspheric intraocular lenses. Ophthalmic Physiology Optics 1991;11:137-146.

42. Spear P. Neural bases of visual deficits during aging. Vis Res 1993;33:2589-2609.

43. Panda-J onas S, J onas J, J akobczyk-Zmija M. Retinal photoreceptor density decreases with age. Ophthalmology 1995:102:1853-1859.

44. Curcio C. Photoreceptor topography in ageing and age-related maculopathy. Eye 2001;14:376-383.

45. Scialfa CT, Kline DW, Wood PK. Structural modeling of contrast sensitivity in adulthood. J Opt Soc Am A 2002;19: 158-165. 
Running title/Author 